\title{
Control of six-phase voltage source converters using dynamic voltage vectors
}

\author{
Juan J. Aciego, I. Gonzalez-Prieto, M. J. Duran \\ Department of Electrical Engineering \\ E.I.I., Malaga University \\ 29071 Malaga, Spain \\ juanjoseaciego@uma.es, ignaciogp87@gmail.com,mjduran@uma.es
}

\begin{abstract}
The use of a single switching state during the whole sampling period in the current regulation of six-phase voltage source converters (VSCs) inevitably generates undesired parasitic $\boldsymbol{x}$ - $\boldsymbol{y}$ currents. Aiming to solve this problem, the creation of virtual/synthetic voltage vectors (VVs) has been recently proposed to ensure zero average $x$-y voltage production. However, the off-line calculation of VVs makes them static and suboptimal. This paper introduces new approach where the virtual voltages are created on-line within a model predictive control (MPC) based current regulation strategy. Since the selection of the switching states and the dwell times varies each sampling period, the resulting vector are termed dynamic voltage vectors (DVVs). This new concept allows an online optimization of the output voltage production depending on the operating point at the expense of a higher computational cost. Simulation results confirm that six-phase VSCs can be successfully regulated using DVVs in an MPC-based current control scheme.
\end{abstract}

Keywords- Current control, dynamic voltage vectors, multiphase voltage source converter

\section{INTRODUCTION}

Classical topics in three-phase electric drives have been revisited for multiphase systems in the last two decades [1], [2], [3], [4], [5] and some innovative applications that are exclusive for multiphase systems have been proposed [7], [8], [9]. While in some cases it was a mere extension of the threephase procedure, the additional degrees of freedom had to be considered cautiously to avoid degrading the drive performance. Specifically, the appearance of parasitic $x-y$ currents in distributed-winding induction machines required some additional effort at the control stage to preserve the efficiency and current quality [5].

In standard field-oriented control (FOC) the regulation of the secondary $x-y$ currents was achieved with the inclusion of some additional proportional-integer (PI) controllers [10], [11]. Unfortunately, the standard version of direct torque control (DTC) and model predictive control (MPC) could not follow this path because they apply a single switching state during the whole sampling period [2]. This fact condemned DTC and MPC to have a significant amount of $x-y$ currents when the stator leakage inductance is small.

The performance degradation of DTC and MPC was improved with the inclusion of virtual/synthetic voltages vectors (termed VVs in what follow) [12]-[17]. The main concept of VVs is essentially to combine two or more active voltage vectors with such a proportion that the average $x-y$ voltage becomes zero [13]. This procedure ensures that $x-y$ currents are limited and, additionally, provides a fault-tolerant capability with a higher degree of simplicity [18]. By definition, the VVs are calculated off-line and consequently they can be referred as static in the sense that the switching states and their proportion is constant. In spite of the suitable performance of VVs both in pre- and post-fault situations, they have some limitations:

L1) They do not optimize the voltage according to the operating point.

L2) Their use is limited to cases when the $x-y$ currents need to be regulated to zero.

L3) They do not fully exploit the utilization of the dc-bus voltage.

Limitation L1 directly derives from the static nature of the VVs. Since they are calculated off-line, they cannot adjust their performance at different instants. Consequently, VVs are simple and computationally efficient at the expense of achieving a suboptimal solution. It may occur that in some cases VVs are the optimal choice, but in other instants it can be better to combine other active vectors, an active vector plus a zero vector or a different proportion of the dwell times.

This work suggests the use of dynamic voltage vectors (DVVs) that are generated online within the model predictive control (MPC) strategy for the current regulation of the multiphase VSC. Both the selection of the switching states and the determination of the dwell times are recalculated each sampling period. Consequently, the voltage vectors and their times of application become variable. Because of this capability to adjust the average output voltage from one sampling period to the next one, the resulting voltage vector is regarded as dynamic in the definition of the term DVV.

With a proper selection procedure in the combination of voltage vectors, DVVs can theoretically overcome limitations L1 to L3 and enhance the control performance at the expense of a higher computational cost. The concept of DVVs and the procedure to create them is introduced for the first time in this paper to confirm the potential interest of this new approach for MPC-based current control of VSCs.

The manuscript is organized as follows. Section II describes the six-phase system and section III reviews the background of MPC. Section IV presents the newly proposed DVVs and explains in detail the procedure for their creation. Section V provides simulation results to confirm the goodness of DVVs in six-phase systems and section VI summarizes the main conclusions. 


\section{SIX-PHASE SYSTEM AND R-L LOAD}

\section{A. Six-phase system generalities}

The system implemented in this paper includes a six-phase VSC using two parallel three-phase inverters. Vector $[S]=\left\{S_{a 1}, S_{b 1}, S_{c 1}, S_{a 2}, S_{b 2}, S_{c 2}\right\}$ describes $2^{6}=64$ avaible VSC switching states. Each vector $[S]$ component represents the switching state in its corresponding VSC leg ( 0 if lower switch is ON and the upper switch OFF and 1 otherwise). A simplified scheme of the implemented topology that includes a $\mathrm{R}-\mathrm{L}$ load is shown in Fig. 1.

In order to determine the phase voltages, transformation from leg to phase values and dc-link voltage $\left(V_{d c}\right)$ are employed as follows:

$\left[\begin{array}{l}v_{a 1} \\ v_{b 1} \\ v_{c 1} \\ v_{a 2} \\ v_{b 2} \\ v_{c 2}\end{array}\right]=\frac{V_{d c}}{3}\left[\begin{array}{rrrrrr}2 & -1 & -1 & 0 & 0 & 0 \\ -1 & 2 & -1 & 0 & 0 & 0 \\ -1 & -1 & 2 & 0 & 0 & 0 \\ 0 & 0 & 0 & 2 & -1 & -1 \\ 0 & 0 & 0 & -1 & 2 & -1 \\ 0 & 0 & 0 & -1 & -1 & 2\end{array}\right] \cdot[S]^{T}$

Vector space decomposition (VSD) provides simpler means for the regulation of multiphase systems and for the prediction of futures switching states. Hence, using the power invariant decoupling Clarke transformation, it is possible to obtain the vector space decomposed voltages.

$[T]=\frac{1}{\sqrt{3}}\left[\begin{array}{cccccc}1 & -1 / 2 & -1 / 2 & \sqrt{3} / 2 & -\sqrt{3} / 2 & 0 \\ 0 & \sqrt{3} / 2 & -\sqrt{3} / 2 & 1 / 2 & 1 / 2 & -1 \\ 1 & -1 / 2 & -1 / 2 & -\sqrt{3} / 2 & \sqrt{3} / 2 & 0 \\ 0 & -\sqrt{3} / 2 & \sqrt{3} / 2 & 1 / 2 & 1 / 2 & -1 \\ 1 & 1 & 1 & 0 & 0 & 0 \\ 0 & 0 & 0 & 1 & 1 & 1\end{array}\right]$

$\left[v_{\alpha}, v_{\beta}, v_{x}, v_{y}, v_{0+}, v_{0-}\right]^{T}=[T]\left[v_{a 1}, v_{b 1}, v_{c 1}, v_{a 2}, v_{b 2}, v_{c 2}\right]^{T}$

Thereby, using (1) and (2), the model of the R-L load included in this multiphase scheme can be expressed into VSD variables (3).

$v_{\alpha}=\left(R_{s}+L_{s} \frac{d}{d t}\right) i_{\alpha}$

$v_{\beta}=\left(R_{s}+L_{s} \frac{d}{d t}\right) i_{\beta}$

$v_{x}=\left(R_{s}+L_{s} \frac{d}{d t}\right) i_{x}$

$v_{s}=\left(R_{s}+L_{s} \frac{d}{d t}\right) i_{y}$

The zero-sequence currents $\left(0_{+} 0_{-}\right)$are omitted from the analysis because the two three-phase R-L loads have isolated neutral points. It is however of paramount importance to properly regulate the $\alpha-\beta$ and $x-y$ currents.

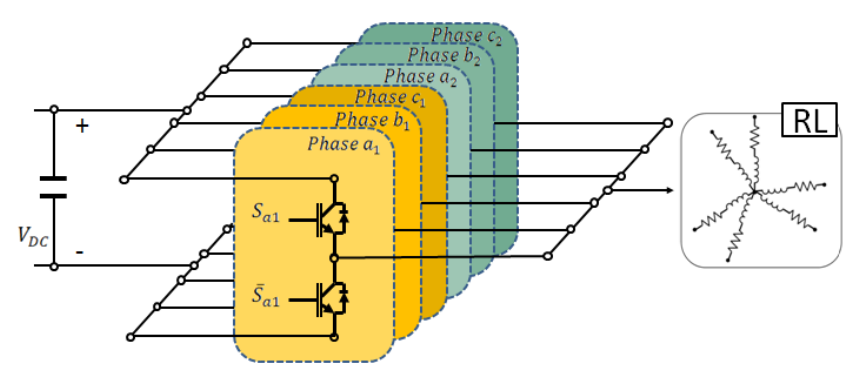

Fig. 1. Scheme of a six-phase system with R-L load.

\section{B. Voltage vectors in a six-phase VSC.}

Applying a single switching state during the whole sampling period (as it is done in MPC) it is not possible to fulfill $\alpha-\beta$ and $x-y$ requirements because each switching state is mapped (Fig. 2) in both planes.

Observing in detail the $2^{6}=64$ voltage vectors mapped in $\alpha-\beta$ and $x-y$ subspaces (Fig. 1), it is possible to group them into small, medium, medium-large and large vector according to their size in the $\alpha-\beta$ subspace. Special attention should be paid to the fact that medium-large vectors in $\alpha-\beta$ subspace result in medium-large in $x-y$ subspace, whereas large vectors in the main $\alpha-\beta$ plane correspond to small vectors in the secondary subspace, and vice versa.

\section{Dynamic virtual voltage vectors}

Since the $2^{6}=64$ switching states available in a six-phase VSC are related by its size and direction in the other subspace, it is possible to obtain an optimal couple of vectors combination and their application times during the sampling period.

Therefore, in order to compose a dynamic voltage vector $\mathrm{DVV}_{\mathrm{i}}$, it is necessary to select the optimal couple of voltage vectors $V_{J}{ }^{1}$ and $V_{J}{ }^{2}$ applied during a time $t_{1}$ and $t_{2}=1-t_{1}$ (in per unit values), respectively. These voltage vectors are selected among the optimal and better three suboptimal switching states that satisfy the requirements in $\alpha-\beta$ and $x-y$ subspaces.
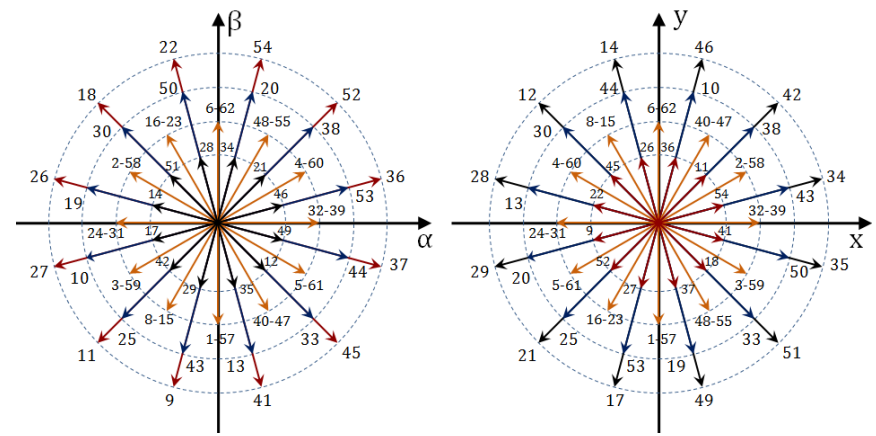

Fig. 2. Voltage vectors in a six-phase VSC. 


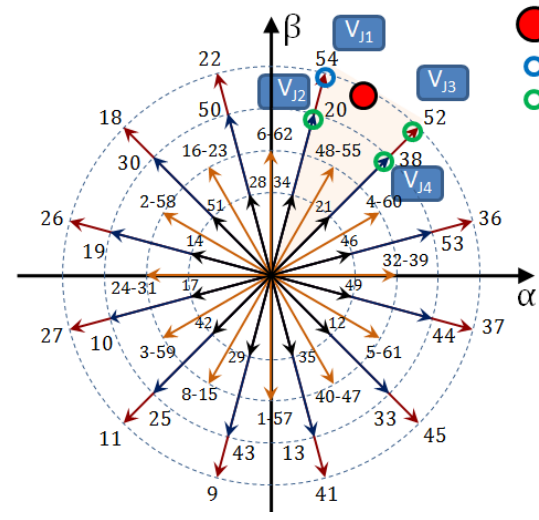

$\alpha \beta$ plane requiriment

Selected voltage vectors

Fig. 3. Four possible selected voltage vectors

For example, to synthesize a DVVi to reach the required $\alpha-\beta$ voltage with minimum copper losses (Fig. 3), it is necessary to select the 4 better voltage vectors $\left(\mathrm{V}_{\mathrm{J} 1}, \mathrm{~V}_{\mathrm{J} 2}, \mathrm{~V}_{\mathrm{J} 3}\right.$ and $\mathrm{V}_{\mathrm{J} 4}$ ) among all available switching states. Then different combinations of this group of vectors are taken two at a time in order to determine the most appropriate couple to achieve a zero average voltage in the $x-y$ plane. This pair of voltage vectors are termed $V_{J^{\prime}}^{1}$ and $V_{J^{\prime}}^{2}$. In order to obtain the application times, different fractions of the sampling period are tested by means of several iterations.

The general expression of the proposed DVVs is:

$$
D V V_{i}=t_{1} \cdot V_{J^{\prime}}^{1}+\left(1-t_{1}\right) \cdot V_{J^{\prime}}^{2}
$$

\section{MODEL PREDICTIVE CONTROL IN SIX PHASE}

MPC schemes have been recently proposed for the control of electric systems. This type of control offers an easy mean to deal with restrictions in cost functions and a fast-current tracking.

In the case of multiphase systems, a disadvantage in standard MPC schemes is however the application of a single switching state during the whole sampling period. This feature is not desirable in multiphase systems due to the impossibility to simultaneous generate the desired voltage in $\alpha-\beta$ and $x-y$ planes. Consequently, it is not possible to provide null values of the $x-y$ currents and the efficiency of the system is spoiled.

MPC of the VSC using DVVs (termed DVV-MPC in what follows) approaches the problem using an adequate selection of voltage vectors and optimal dwell times in a single sampling period. The objective of the current controller is to track the reference VSD currents $i_{\alpha \beta x y}^{*}$. Using a discrete model of the system, the future behavior of the output variable $\hat{i}_{\alpha \beta x y}$ can be estimated. Based on the VSD approach and Clarke transformation [T], the predictive model can be expressed as follows:

$$
\frac{d}{d t}\left[X_{\alpha \beta x y}\right]=[A] \cdot\left[X_{\alpha \beta x y}\right]+[B] \cdot\left[U_{\alpha \beta x y}\right]
$$

where:

$$
\begin{aligned}
& {\left[\begin{array}{ll}
U_{\alpha \beta x y}
\end{array}\right]=\left[\begin{array}{lll}
V_{\alpha} & V_{\beta} & V_{x} \\
V_{y}
\end{array}\right]^{T}}
\end{aligned}
$$

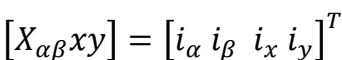

The matrices $[A]$ and $[B]$ define the R-L load and its coefficients are related with their parameters. This work uses the standard Euler discretization to compute the predictive model.

There are three optimization steps included in the DVVMPC to provide optimal switching states and their corresponding application times (see Fig. 4). These optimization steps select the optimal gating signal in order to minimize a cost function $J_{i}$. The latter is computed for all available switching states and takes in to account the error in both $\alpha-\beta$ and $\mathrm{x}-\mathrm{y}$ subspaces.

$J=K_{\alpha} * e_{\alpha}^{2}+K_{\beta} * e_{\beta}{ }^{2}+K_{x} * e_{x}^{2}+K_{y} * e_{y}{ }^{2}$

where:

$e_{\alpha}=\left(i_{\alpha}^{*}-\hat{\imath}_{\alpha}\right)$

$e_{\beta}=\left(i_{\beta}^{*}-\hat{i}_{\beta}\right)$

$e_{\alpha}=\left(i_{\alpha}^{*}-\hat{\imath}_{\alpha}\right)$

$e_{\beta}=\left(i_{\beta}^{*}-\hat{\imath}_{\beta}\right)$

where coefficients $K_{\alpha}, K_{\beta}, K_{x}, K_{y}$ are the weighting factors for each component (see Table II). The value of these coefficients must be selected according to the control objectives.

\section{DVV-MPC PROPOSED CONTROL ALGORITHM}

The proposed control algorithm attempts to be an online procedure to compose a synthetic vector in order to satisfactorily obtain the $\alpha-\beta$ current tracking with minimum cooper losses. These latter copper losses require an adequate $x-y$ current control

For this purpose, a three stages algorithm is proposed (Fig. 4 and Fig. 5). In a first step (stage 1), the optimum voltage vector and three following suboptimum are selected, in order to satisfy the mentioned control objectives. In the second step (stage 2), the better combination of these four preselected voltage vectors is determined. Further, in this stage, it is intensified the importance of minimizing the $x-y$ current error

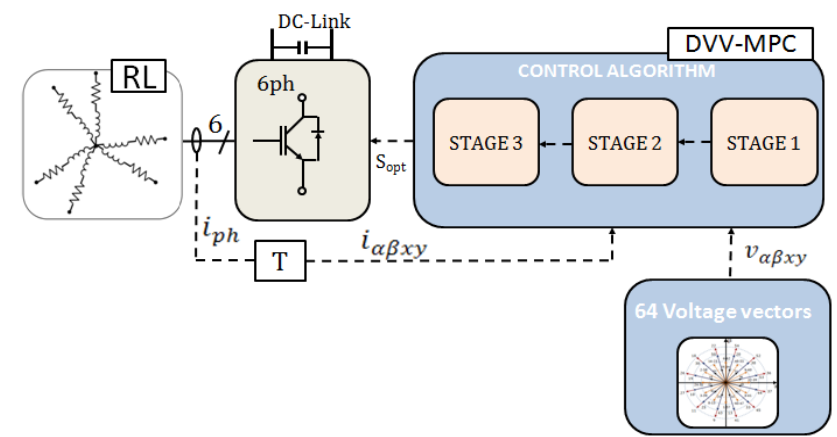

Fig. 4. Proposed DVV-MPC control scheme 
via the addition of a new term. Thus, two voltage vectors are definitely selected. In the third stage, stage 3 , it is obtained the application times for the two selected voltage vectors. More details of the proposed control method can be found below:

Stage 1 . In this stage the four better voltage vectors are selected. Its performance matches with a conventional MPC. The set of voltage vectors for a six-phase converter is $2^{6}$ so the algorithm iterates 64 times, obtaining the same number of cost functions. The lower value of $J_{m}$, the better is the performance control attending to both objectives in $\alpha-\beta$ and $x-y$ frames. Therefore, the optimum voltage vector and the following three better correspond to $J_{1}, J_{2}, J_{3}, J_{4}$. These latter four are combined in the following stage.

Stage 2. This stage allows to define a new type of cost function $\left(J^{\prime}\right)$. These latter are composed by the combination of the group $J_{1}, J_{2}, J_{3}, J_{4}$ taken two at a time and an added term minimizing the components in the secondary subspace and being then chosen the optimum combination. Cost functions $\left(J_{m-n}^{\prime}\right)$ are six $\left(J_{1-2}^{\prime}, J_{1-3}^{\prime}, J_{1-4}^{\prime}, J_{2-3}^{\prime}, J_{2-4}^{\prime}, J_{3-4}^{\prime}\right)$ and the lower is the value of $J_{n}^{\prime}$, the better is the control performance in both subspaces. The optimum value $\left(J_{o p t}^{\prime}\right)$ allows obtaining the two selected voltage vectors to be used in the next stage $\left(V_{J},{ }^{1}\right.$ and $V_{J^{2}}{ }^{2}$ ). For example, $J_{1-2}^{\prime}$ is determined as follows:

$$
\begin{aligned}
& J_{1-2}^{\prime}=J_{1}+J_{2}+k_{w} *\left[\left(v_{x}^{1}+v_{x}^{2}\right)^{2}+\left[\left(v_{y}^{1}+v_{y}^{2}\right)^{2}\right]\right. \\
& \text { where: }
\end{aligned}
$$

$K_{w}$ : weighting factor for term that penalizes copper losses (see Table II).

\section{$v_{x}^{i}$ : voltage vector i horizontal component \\ $v_{y}^{i}$ : voltage vector i vertical component}

$J_{i:}$ Each one of the cost function corresponding to the preselected voltage vectors in stage $1, i \in\{1,2,34\}$.

The added third term, in order to emphasize the importance of the minimum copper losses in the cost function, evaluates the $x-y$ vector addition in every sampling time including the weighting factor $K_{w}$. Null vector (V0) is included as the fourth selected voltage vector when the latter has not been chosen in previous stage.

Stage 3. So as to synthesize the optimal virtual vector, it is necessary to determine the application times for each of the selected voltage vectors $\left(V_{J}{ }^{1}\right.$ and $\left.V_{J^{2}}{ }^{2}\right)$. These application times $\left(t_{1}\right.$ and $\left.t_{2}\right)$ are expressed in per unit taking $T_{S}$ as base value. The first selected voltage vector $\mathrm{V}_{\mathrm{J}},{ }^{1}$ is applied during time $\mathrm{t}_{1}$ and $V_{J}{ }^{2}$ is applied for the rest of the sampling period $\left(t_{2}\right)$. Therefore, the algorithm iterates 9 times in stage 3, sweeping from 0.55 to 0.95 and obtaining the same number of cost functions and associated dwell times.

It is worth explaining that values of $t_{1}$ from 0.55 to 0.95 have been considered because this application times correspond to the optimal voltage vector and must be applied more than the second one. Moreover, a virtual vector is composed by at least two voltage vectors and application times above $0.95 \%$ of $\mathrm{T}_{\mathrm{s}}$ (see Table I) entails a single switching state during the whole sampling period.

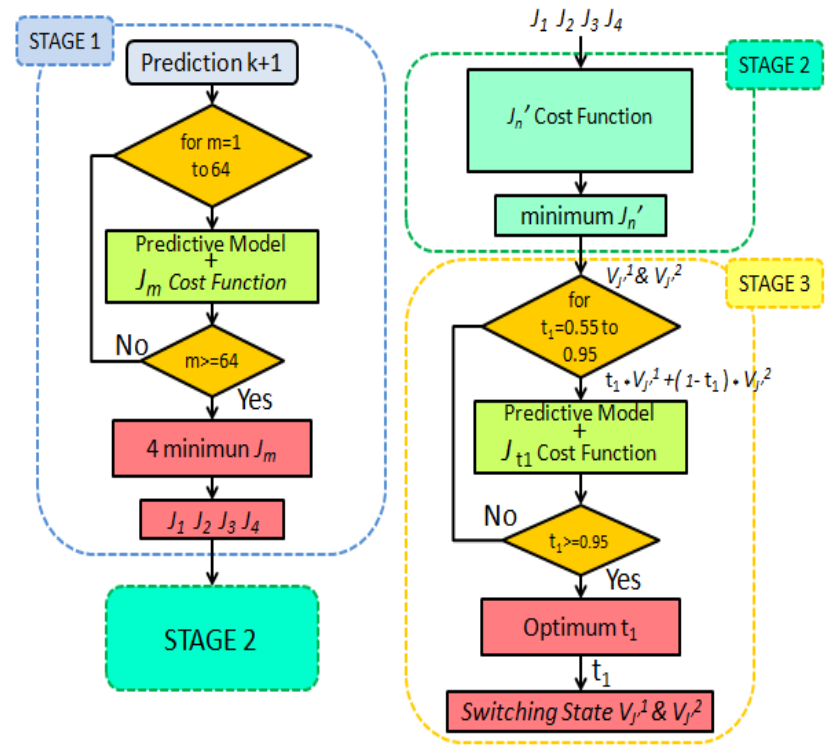

Fig. 5. Flowchart proposed DVV-MPC algorithm

\section{SIMULATION RESULTS}

An assessment of the proposed DVV-MPC has been realized in this section. In practice, three different operation situations have been simulated. For that purpose, the six-phase $R-L$ load and the developed control scheme have been implemented in Matlab/Simulink. Simulation and system parameters have been summarized in Table I. On the other hand, Table II presents the weighting factors selected for these tests.

\section{A. Test 1}

Test 1 evaluates the performance of the proposed DVVMPC in a stationary situation when the $\left|i_{\alpha \beta}^{*}\right|=4 A$. As shown in Fig. 6. (left plots), the tracking of $\alpha-\beta$ currents is satisfactorily performed. In fact, the THD of the $\alpha$ current for this operation point is $1.22 \%$. This low value of the $\alpha$ current THD allows to validate the $\alpha-\beta$ current regulation at this operating point. However, the goodness of the phase currents is also dependent on the $x$-y current regulation. Therefore, a low value of the $x$ $y$ currents is necessary to provide acceptable phase currents. The obtained $x-y$ currents have been depicted in Fig. 6.b (left and right plots). As shown in Fig 6.b, $x-y$ currents are close to zero and therefore the phase currents present a low ripple value (see Fig. 6.c). In order to quantify the quality of the phase current the THD has been estimated for the phase $a_{1}$ (THD=1,84\%).

TABLE I

SIMULATION AND SYSTEM PARAMETERS

\begin{tabular}{|c|c|}
\hline$T_{s}$ & $100 \mu \mathrm{s}$ \\
\hline$R_{s}$ & $8 \Omega$ \\
\hline$L_{s}$ & $110 \mathrm{mH}$ \\
\hline$V_{d c}$ & $300 \mathrm{~V}$ \\
\hline
\end{tabular}


TABLE II

WEIGTHING FACTORS

\begin{tabular}{|c|c|}
\hline$k_{\alpha \beta}$ & 1 \\
\hline$k_{x y}$ & 0.5 \\
\hline$k_{w}$ & $10^{-5}$ \\
\hline$k_{\alpha \beta 1}$ & 1 \\
\hline$k_{x 1 y 1}$ & 0.3 \\
\hline
\end{tabular}

\section{B. Test 2}

To validate the DVV-MPC behavior in a different stationary situation a lower value of the $\left|i_{\alpha \beta}^{*}\right|$ has been selected in the test $2\left(\left|i_{\alpha \beta}^{*}\right|=0.7\right.$ in this case $)$. Although the operation conditions are completely different for this new scenario compared to the conditions of test 1 , the regulation of $\alpha-\beta$ and $x-y$ currents is again good (see Fig. 6.a and Fig. 6.b). To illustrate this fact from another point of view, Fig. 6.c shows $\alpha-\beta$ and $x-y$ current diagrams, where the $\alpha-\beta$ current diagram presents a circle shape with a low ripple. Due to the suitable regulation of $\alpha-\beta$ and $x-y$ currents, the obtained phase currents show a satisfactory waveform with a reduced harmonic distortion. In this case the THD of the phase $a_{1}$ current is $10,08 \%$.

\section{C. $\quad$ Test 3}

Finally, the dynamic response of proposed control scheme has been tested in test 3 . For that purpose, a step in the reference value of the $\left|i_{\alpha \beta}^{*}\right|$ has been included at $t=0,7 \mathrm{~s}$. Specifically, $\left|i_{\alpha \beta}^{*}\right|$ is equal to $5 \mathrm{~A}$ in the first stage of the test and $\left|i_{\alpha \beta}^{*}\right|=3.5 \mathrm{~A}$ after $t=0.7 \mathrm{~s}$. As expected, the regulation of the $x-y$ currents is independent from the transient situation and their values are closed to zero during the whole test (Fig. 7.b). However, $\alpha-\beta$ currents need to change their values according to the introduced reference step (Fig. 7.a). Although an instantaneous reduction of the $\left|i_{\alpha \beta}^{*}\right|$ has been added, the tracking of the reference value is successful, as shown in Fig. 7.a. Notice that this satisfactory dynamic response has been obtained without degradation of the quality of the phase currents (Fig. 7c).

\section{CONCLUSIONS}

The use of static virtual voltage vectors is a simple and cost-effective solution for the current regulation of six-phase VSCs. However, their incapability to adjust the output voltage production to different operating points makes VVs a suboptimal choice. Following an alternative path, it is possible to calculate the virtual voltage vectors online. Although the computational cost inevitably increases, the three-stage procedure that is proposed in this work allows the inclusion of dynamic voltage vectors with only 9 additional iterations in the MPC scheme. It is found however that DVVs provide the flexibility to select different switching states and dwell times, hence overcoming the limitations of static VVs. Simulation results confirm that DVVs can be successfully used within the MPC-based current regulation scheme of a six-phase VSC, providing a satisfactory current tracking and quality.

\section{REFERENCES}

[1] E. Levi, F. Barrero and M.J. Duran, "Multiphase machines and drives Revisited," IEEE Trans. Ind. Electron., vol. 63, no. 1, pp. 429-432, Jan. 2016.

[2] Duran, M.J., Levi , E. and Barrero, F., Multiphase Electric Drives: Introduction, Wiley Encyclopedia of Electrical and Electronics Engineering, pp. 1-26, 2017.

[3] E. Levi, F. Barrero and M.J. Duran, "Multiphase machines and drives Revisited,” IEEE Trans. Ind. Electron., vol. 63, no. 1, pp. 429-432, Jan. 2016.

[4] Ayman S., Abdel-Khalik, Ahmed M. Massoud, Shehab Ahmed, "Application of Standard Three-Phase Stator Frames in Prime Phase Order Multiphase Machine Construction," IEEE Transactions on Industrial Electronics, vol. 66, no. 4. pp. 2506 - 2517, 2019.

[5] Amir Negahdari, Alejandro G. Yepes, Jesus Doval-Gandoy and Hamid A. Toliyat, " Efficiency Enhancement of Multiphase Electric Drives at Light-Load Operation Considering Both Converter and Stator Copper Losses," IEEE Transactions on Power Electronics, vol. 34, no. 2, pp. 1518 - 1525. 2019.

[6] Mohamed I. Daoud, Ahmed A. Elserougi , Ahmed M. Massoud, Radu Bojoi, Ayman S. Abdel-Khalik and Shehab Ahmed, " Zero-/LowSpeed Operation of Multiphase Drive Systems With Modular Multilevel Converters," IEEE Access, vol. 7, pp. 14353 - 14365.

[7] Mario J. Duran, Ignacio Gonzalez-Prieto, Federico Barrero, Emil Levi, Luca Zarri, Michele Mengoni,"A Simple Braking Method for SixPhase Induction Motor Drives With Unidirectional Power Flow in the Base-Speed Region," IEEE Transactions on Industrial Electronics, vol. 64, no. 8, pp. 6032-6041.

[8] Ivan Subotic, Nandor Bodo and Emil Levi, "Single-Phase On-Board Integrated Battery Chargers for EVs Based on Multiphase Machines" IEEE Transactions on Power Electronics, vol. 31, no. 9, pp. 6511-6523.

[9] M. Bermudez, I. Gonzalez-Prieto, F. Barrero, H. Guzman, M. J. Duran, and X. Kestelyn, "Open-phase fault-tolerant direct torque control technique for five-phase induction motor drives," IEEE Trans. Ind. Electron., vol. 61, no. 9, pp. 4474-4484, Sep. 2014.

[10] Hang Seng Che, Emil Levi, Martin Jones, Wooi-Ping Hew and Nasrudin Abd. Rahim, " Current Control Methods for an Asymmetrical Six-Phase Induction Motor Drive," IEEE Transactions on Power Electronics, vol. 29, no. 1, pp. 407 - 417. 2014.

[11] A. Tenconi, S. Rubino and R. Bojoi, " Model Predictive Control for Multiphase Motor Drives - a Technology Status Review," International Power Electronics Conference (IPEC-Niigata 2018 -ECCE Asia, pp.732-739.

[12] Libo Zheng, John E. Fletcher, Barry W. Williams and Xiangning He, "A Novel Direct Torque Control Scheme for a Sensorless Five-Phase Induction Motor Drive," IEEE Transactions on Industrial Electronics, vol. 58, no. 2,pp. 503-513. 2011

[13] I. Gonzalez-Prieto, M.J. Duran, Juan J. Aciego, C. Martin and F. Barrero "Model predictive control of six-phase induction motor drives using virtual voltage vectors," IEEE Trans. on Ind. Electron., vol. 65, no. 1, pp. 27-37, 2018.

[14] Juan J. Aciego, I. González Prieto and M. J. Duran, "Model Predictive Control of Six-Phase Induction Motor Drives Using Two Virtual VoltageVectors," IEEE Journal of Emerging and Selected Topics in Power Electronics, vol. 7, no. 1, pp. 321-330.

[15] J.K. Pandit, M.V. Aware, R.V. Nemade and E. Levi, "Direct torque control scheme for a six-phase induction motor with reduced torque ripple," IEEE Transactions on Power Electronics, vol. 32, pp.7118$7129,2017$.

[16] M.R. Arahal, F. Barrero, S. Toral, M.J. Duran, and R. "Multiphase current control using finite-state model-predictive control," Control Eng. Pract., vol. 17, no. 5, pp. 579-587, 2009.

[17] Y. Zhang and H. Yang, "Two-vector-based model predictive torque control without weighting factors for induction motor drives," IEEE Trans. Power Electron., vol. 31, no. 2, pp. 1381-1390, Feb. 2016.

[18] I. Gonzalez-Prieto, M. J. Duran, M. Bermúdez, F. Barrero and C. Martín, "Assessment of Virtual-Voltage-based Model Predictive Controllers in Six-phase Drives under Open-Phase Faults," IEEE Journal of Emerging and Selected Topics in Power Electronics., to be published., doi:10.1109/JESTPE.2019.2915666 
a)

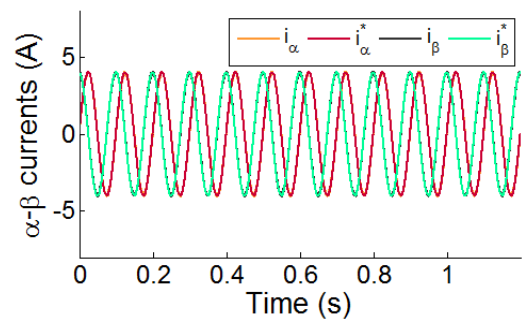

b)

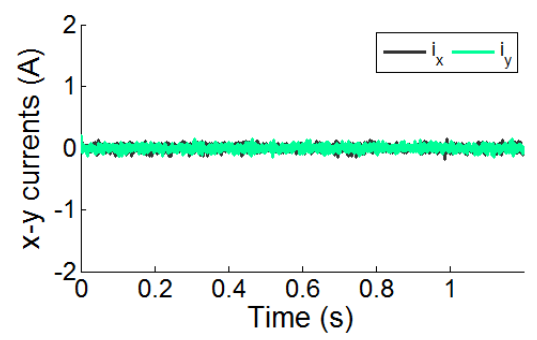

c)

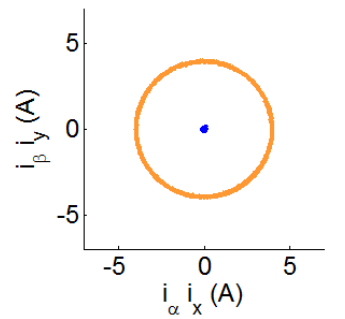

d)

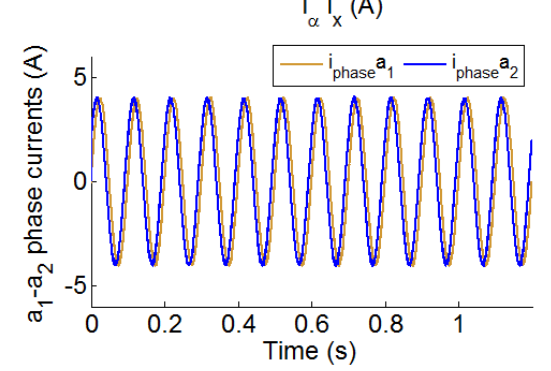

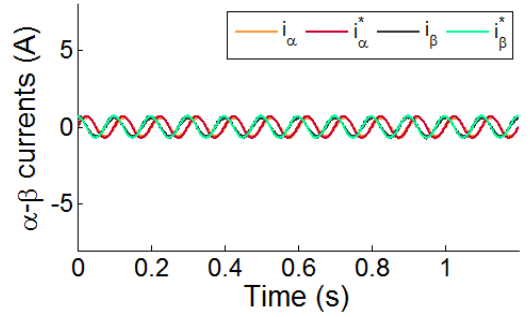
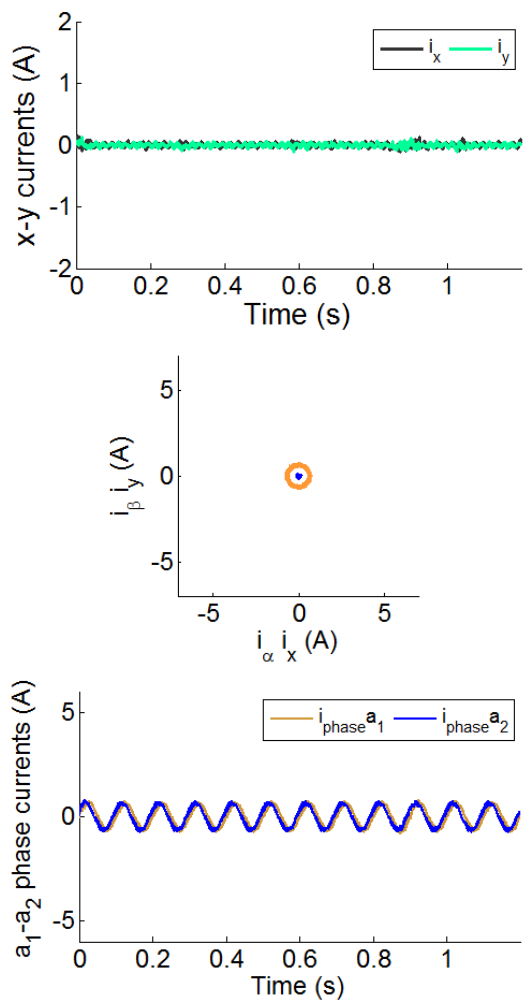

Fig. 6. Tests for DVV-MPC in high $\alpha-\beta$ requirements point (left plots) and low $\alpha-\beta$ requirements point (right plots). From top to bottom: a) $\alpha-\beta$ currents, b) $x-y$ currents, c) $\alpha-\beta$ and $x-y$ currents and d) phase currents.
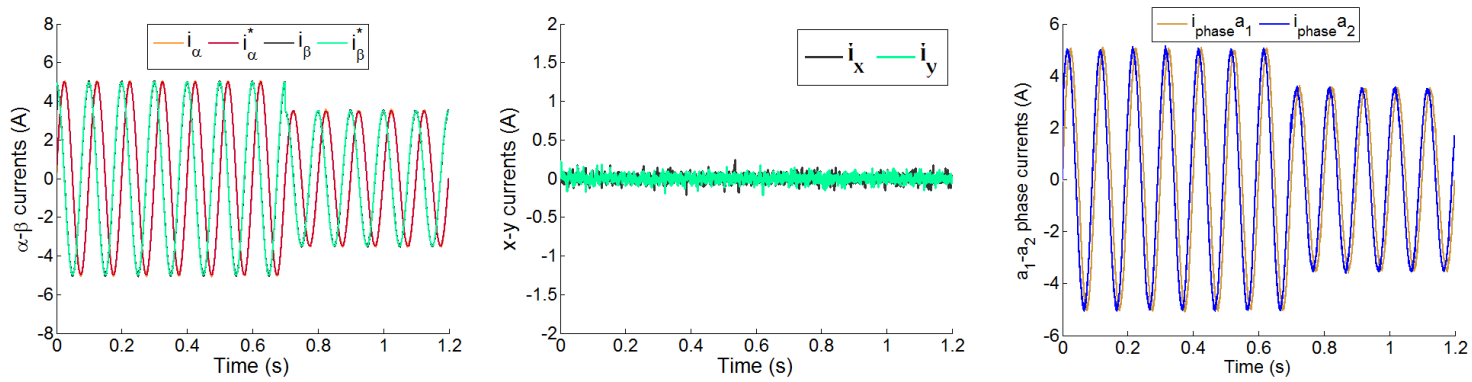

Fig. 7. Test dynamic response for a $\alpha-\beta$ requirements reference change (step). From left to right: $\alpha-\beta$ currents, $\mathrm{x}-\mathrm{y}$ currents and phase currents. 